\title{
Change not State: Perceptual coupling in multistable displays reflects transient bias induced by perceptual change
}

\author{
Alexander Pastukhov ${ }^{1,2}$ - Claus-Christian Carbon ${ }^{1,2}$
}

Accepted: 20 May 2021 / Published online: 2 August 2021

(C) The Author(s) 2021

\begin{abstract}
We investigated how changes in dynamic spatial context influence visual perception. Specifically, we reexamined the perceptual coupling phenomenon when two multistable displays viewed simultaneously tend to be in the same dominant state and switch in accord. Current models assume this interaction reflecting mutual bias produced by a dominant perceptual state. In contrast, we demonstrate that influence of spatial context is strongest when perception changes. First, we replicated earlier work using bistable kinetic-depth effect displays, then extended it by employing asynchronous presentation to show that perceptual coupling cannot be accounted for by the static context provided by perceptually dominant states. Next, we demonstrated that perceptual coupling reflects transient bias induced by perceptual change, both in ambiguous and disambiguated displays. We used a hierarchical Bayesian model to characterize its timing, demonstrating that the transient bias is induced 50-70 ms after the exogenous trigger event and decays within $\sim 200-300 \mathrm{~ms}$. Both endogenous and exogenous switches led to quantitatively and qualitatively similar perceptual consequences, activating similar perceptual reevaluation mechanisms within a spatial surround. We explain how they can be understood within a transient selective visual attention framework or using local lateral connections within sensory representations. We suggest that observed perceptual effects reflect general mechanisms of perceptual inference for dynamic visual scene perception.
\end{abstract}

Keywords Visual perception $\cdot$ Multistability $\cdot$ Perceptual coupling $\cdot$ Switch transient $\cdot$ Selective attention

Perception is the prerequisite of all cognitive acts and it must deliver a singular, determinate percept that represents the outside world despite intrinsically noisy and ambiguous sensory inputs. To solve this underdetermined problem, it strongly relies on additional sources of information, such as spatial context (Todorović, 2010). The latter has been extensively studied, and it has a profound effect on the perception of size (Murray et al., 2006), color (Hansen et al., 2007), or tilt (Gibson \& Radner, 1937), to name just a few dimensions. The spatial context also strongly affects the perception of multistable stimuli, such as binocular rivalry or kinetic-depth effect (KDE; see Movie S1). These displays are perceptually

Alexander Pastukhov

pastukhov.alexander@gmail.com

1 Department of General Psychology and Methodology, University of Bamberg, Bamberg, Bavaria, Germany

2 Research Group EPÆG (Ergonomics, Psychological Æsthetics, Gestalt), Bamberg, Bavaria, Germany unstable and give rise to two (or more) distinct interpretations so that observers' perception endlessly alternates between them for as long as they view the stimulus. For these displays, an unambiguous static context alters switching dynamics by changing the balance of the perceptual competition, prolonging the dominance of the favored perceptual interpretation and curtailing that for the other one (Fang \& He, 2004; Intaite et al., 2013; Klink et al., 2012; Sereno \& Sereno, 1999).

The multistable displays are also an excellent tool for studying dynamic contexts. When several such displays are viewed simultaneously, their perception tends to be coupled together, so they are likely to be in the same dominant perceptual state and switch to a new state in accord (Attneave, 1968; Eby et al., 1989; Ramachandran \& Anstis, 1983, 1985). This perceptual coupling reveals the mutual influence of individual multistable objects, with each one serving both as perceptual context and as a perceptual probe. The strength of perceptual coupling depends on the similarity of the bistable property (e.g., the orientation of the axis of rotation for KDE), objects' proximity (smaller distances lead to stronger coupling), and 
ambiguity (disambiguation of one of the objects diminishes coupling (Grossmann \& Dobbins, 2003; Pastukhov et al., 2018; but see Freeman \& Driver, 2006; Klink et al., 2009). It is hypothesized to work via either a top-down mechanism (Grossmann \& Dobbins, 2003) or local lateral connections (Klink et al., 2009). Its primary purpose is assumed to provide positive feedback to stabilize perception under challenging conditions such as multistablity.

Importantly, the perceptual coupling is thought to reflect a bias produced by a dominant perceptual state that facilitates the dominance of that same state in a neighboring object (Grossmann \& Dobbins, 2003; Klink et al., 2009). However, as noted above, the perceptual coupling is reduced when one of the objects is disambiguated (Grossmann \& Dobbins, 2003; Pastukhov et al., 2018). This creates a contradiction: If perceptual coupling reflects the influence of dominant perceptual states, the exogenous bias should stabilize the exogenously biased state, prolonging its dominance and enhancing rather than curtailing the perceptual coupling. Similarly, such state-based stabilizing feedback should slow down perceptual alternations, yet the perceptual coupling leads to faster switches (Pastukhov et al., 2018). Taking this into account, we hypothesized that perceptual coupling could be better explained by a transient mechanism that is activated by a change in perception rather than by its state and, therefore by the dynamics of the perceptual context. Below, we reexamine the phenomenon by replicating earlier work and determining that perceptual coupling cannot be explained by the influence of stable perceptually dominant states. Next, we demonstrate that coupling relies on transient interaction induced by perceptual switches that trigger a reevaluation of perception and produce transient bias favoring the new perceptual state in nearby spatial locations and objects. Finally, we show that this transient bias is a general consequence of perceptual changes as it is evoked by perceptual switches in both ambiguous and unambiguous displays.

\section{Methods}

\section{Participants}

Twenty-nine participants took part in Experiments 1 and 2, 27 females, two males; age range: 19-41 years. Only 15 people did experiments with coaxial and parallel layouts, whereas five did only the coaxial layout, and nine did only the parallel layout. Fifteen participants took part in Experiment 3, 12 females, three males; age range: 1835 years. Two of the participants completed only two out of three experimental sessions, and three completed only one experimental session. These five participants were excluded from further analysis.

\section{Apparatus}

Displays were presented on a Samsung SyncMaster 2233RZ monitor that had $47.5 \times 29.5 \mathrm{~cm}$ visible area, with a resolution of 1,680 $\times 1,050$ pixels and a refresh rate of $120 \mathrm{~Hz}$. The viewing distance was $50 \mathrm{~cm}$. Participants responded using a Cedrus RB-530 response box. Stimuli were generated using custom code and the PsychoPy library (Peirce et al., 2019).

\section{Displays}

For a video example of stimuli for all experiments, please refer to Movie S1 in the Supplementary Materials.

Kinetic-depth effect (KDE) displays were an orthographic projection of a sphere (diameter $3.5^{\circ}$ ) that rotated around the vertical axis at the speed of $0.25 \mathrm{~Hz}$. Each sphere consisted of 400 white or yellow semitransparent dots (diameter $0.08^{\circ}$ ) distributed randomly on the surface. The prime sphere in Experiment 3 was an exception, as it consisted of 50 dots to facilitate exogenously triggered reversals. In the parallel layout, the spheres were located to the left and the right of fixation $\left( \pm 1.75^{\circ}\right.$, the spheres were touching) so that their axes of rotation were parallel (see Fig. 1a). In the coaxial layout, they were presented above and below the fixation $\left( \pm 1.75^{\circ}\right)$ so that their axes of rotation were coaxial. To facilitate perceptual grouping within a single object, one sphere was colored white, while the other one was yellow (Pastukhov et al., 2018). Spheres alternated their color on every block.

\section{Experiment 1. Continuous presentation}

Experiment 1 consisted of 16 blocks generated from four levels of disambiguation, two locations of the prime (left and right for the parallel layout, above and below for the coaxial layout), and two directions of rotation of the disambiguated prime sphere (left and right).

The direction of rotation of the prime sphere was disambiguated via stereoscopic depth cues (anaglyph presentation). The projections for two eyes differed in their orientation around the vertical axis by $0^{\circ}$ (fully ambiguous), $0.25^{\circ}, 0.5^{\circ}$, or $1^{\circ}$. The strength of the disambiguation cues was constant throughout the entire presentation.

Each block started with a random onset delay (0.7-1.5 s) and lasted for 1 minute. Participants viewed the two spheres and continuously reported on their perceptual states. For the parallel layout, we used the following mapping: left or right button, if both spheres rotated, respectively, to the left or the right; up button, if left sphere rotated to the right and right sphere to the left (described as "into the screen"); down button, 




\section{B) Exp. 2 \& 3}

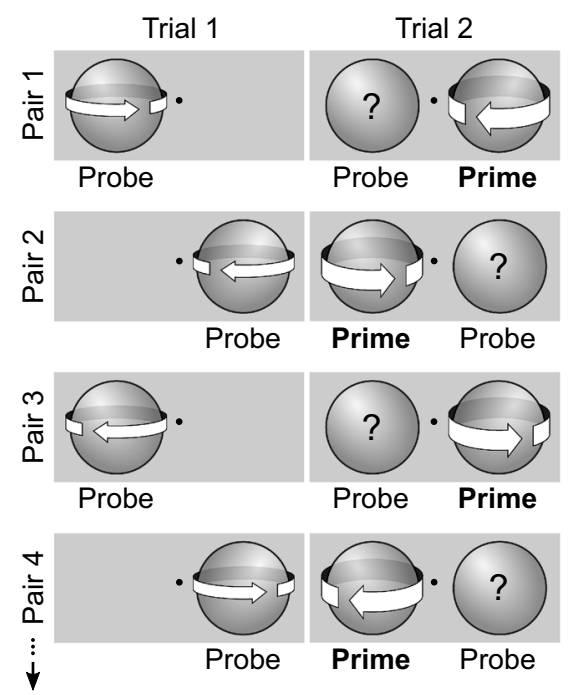

\section{D) Exp. 2}

E) Exp. 3

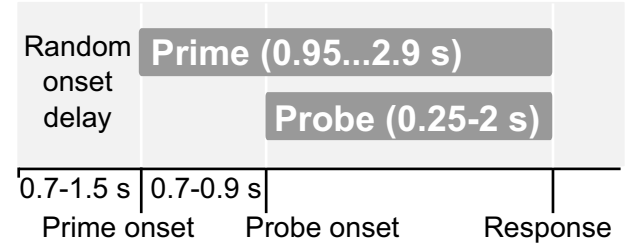

Fig. 1 Stimuli and procedure. a Schematic display. The probe sphere (right) was fully ambiguous (arrows indicate that both directions of rotation were equally dominant), whereas the prime (left) was biased towards one direction of rotation (indicated by a single arrow). b Experiments 2 and 3. A block presentation sequence was split into pairs of trials (rows). In Trial 1, participants reported on the direction of the probe alone. In Trial 2, the prime appeared at the complementary position before the probe and was biased to rotate in the opposite direction to that reported for the probe on Trial 1. c Experiments 2 and 3. For the prime, the strength of the disambiguation cues was maximal at the onset and then linearly reduced to the predefined level over $400 \mathrm{~ms}$. d Experiment 2. Presentation schedule for Trial 2 with both prime and probe spheres. The

if left sphere rotated to the left and right sphere to the right (described as "out of the screen"); the round middle button, if the perception was unclear (approximately $0.2 \%$ of total block time). For the coaxial layout, participants were instructed to press left or right button if both spheres rotated, respectively, to the left or the right; down button whenever they perceived one of the counterrotation states; the round middle button, if the perception was unclear (approximately $0.1 \%$ of total block time).

\section{Experiment 2. Brief asynchronous presentation}

Experiment 2 consisted of eight blocks. Each block consisted of 64 trials with four levels of disambiguation $\left(0^{\circ}, 0.25^{\circ}, 0.5^{\circ}\right.$, or $1^{\circ}$, same as in Experiment 1), four probe durations ( $0.25 \mathrm{~s}$, $0.5 \mathrm{~s}, 1 \mathrm{~s}$, or $2 \mathrm{~s}$ ), and two locations of the prime (left and right

prime appeared before the probe and was biased to rotate in the direction opposite to that reported for the probe on the previous trial. Participants reported on the direction of rotation of both spheres. e Experiment 3. Presentation schedule for the Trial 2 for a switching prime. A prime appeared before the probe and was biased to rotate in the same direction as reported for the probe on the previous trial. An inversion of on-screen motion in the prime sphere triggered a perceptual switch to the opposite direction of rotation. The probe sphere appeared $-300-400 \mathrm{~ms}$ relative to the switch event. The prime disappeared $200 \mathrm{~ms}$ after the onscreen motion inversion. Participants reported on the direction of rotation of the probe sphere

off the fixation, same as in Experiment 1). The onset of each trial was randomly delayed by $0.7-1.5 \mathrm{~s}$.

The block sequence was divided into 32 pairs of trials (see Fig. $1 \mathrm{~b}$ ). During the Trial 1, only a fully ambiguous probe sphere was presented (presentation duration was $1 \mathrm{~s}$, left column in Fig. 1b). Participants reported on its direction of rotation, informing us about the current dominant rotation direction at that location. Prior work showed that an ambiguous probe at this location tends to rotate in the same "default" direction (Knapen et al., 2009) unless its perceptual state is influenced by other factors, such as the dominant state of the prime in our case. During the subsequent Trial 2 (right column in Fig. 1b), the prime sphere appeared at the complementary position before the probe and was strongly biased to rotate in the opposite direction to that reported for the probe on the first trial (see Fig. 1d). The ambiguity of the prime sphere was linearly reduced to the target disambiguation strength 
throughout $400 \mathrm{~ms}$ (see Fig. 1c). Once the probe appeared (stimulus onset asynchrony was 700-900 ms), both were presented for another $0.25 \mathrm{~s}, 0.5 \mathrm{~s}, 1 \mathrm{~s}$, or $2 \mathrm{~s}$ and disappeared simultaneously. Participants reported on the final direction of rotation of both spheres using the same mapping as in Experiment 1 . In the case of probe-only trials, they were instructed to use only left, right, and unclear options. Participants reported unclear perception in approximately $0.4 \%$ (probe only) and $2.1 \%$ (both spheres) of trials for the coaxial layout and in $0.1 \%$ (probe only) and $1.7 \%$ (both spheres) for the parallel layout.

\section{Experiment 3. Brief asynchronous presentation with an on-screen-motion reversal event}

Experiment 3 used only the parallel layout and consisted of three experimental sessions. Each session consisted of 10 blocks with three prime conditions and 10 probe onset times combinations randomly mixed within each block. Session 1 had 20 trials for all three prime conditions (see below) so that ten probe stimulus onset asynchronies (SOA) were presented twice (60 trials in total). Sessions 2 and 3 used only the two switching primes conditions (see below) and had 40 trials per condition (four repetitions of each probe SOA, 80 trials per block). Please note that two participants completed only two sessions, and three participants completed only one session. They were excluded from the analysis, but the incomplete data sets are available at the online repository.

The procedure was similar to Experiment 2 with the same sequence of probe-only and prime-plus-probe pairs of trials (see above and Fig. 1b), but with a different prime presentation schedule (see Fig. 1e). Specifically, we used three prime presentation conditions: (1) switching ambiguous prime, (2) switching biased prime, and (3) stable ambiguous prime (condition identical to Experiment 2). The biased prime (Condition 2 ) remained strongly biased $\left(1^{\circ}\right)$ throughout the entire trial. Ambiguous primes (Conditions 1 and 3) were strongly biased $\left(1^{\circ}\right)$ at the onset, and their disambiguation cues were reduced to zero throughout $400 \mathrm{~ms}$, as in Experiment 2 (see Fig. 1c). Stable ambiguous primes (Condition 3 ) were initially biased to rotate in the direction opposite to that reported for the probe on the previous trial (as in Experiment 2). Switching primes (Conditions 1 and 2) were biased to rotate in the same direction as one reported for the probe on the previous trial so that the opposite direction of rotation would become dominant following a perceptual switch. For switching primes, the 2D on-screen motion was reversed $900-1,100 \mathrm{~ms}$ after the onset leading to a perceptual switch in the direction of rotation (Pastukhov et al., 2012). Probe onset was timed relative to the on-screen motion reversal (a hypothetical one, in case of stable ambiguous primes) with SOAs $-300,-200,-100,-50,0,50,100,200,300$, and $400 \mathrm{~ms}$. Prime disappeared $200 \mathrm{~ms}$ after the probe onset. The probe duration was always $1,000 \mathrm{~ms}$ (i.e., it disappeared $800 \mathrm{~ms}$ after the prime). Participants responded on the final direction of rotation of the probe (left/right/unclear), there were no unclear responses.

\section{Statistical analysis}

Statistical analysis was performed in R, Version 4.0.0 (R Core Team, 2019) using tidyverse packages (Wickham et al., 2019).

For Experiments 1 and 2, we quantified the importance of individual fixed effects using the following measures. For each fixed effect term (referred to as $\beta$ in figures), we computed its estimated mean value and a $95 \%$ credible interval, a range that contains $95 \%$ of values from the sampled posterior distribution (CI, also called compatibility interval). Additionally, each term's importance was assessed by fitting a full model and the model without a term (a drop-one approach) and comparing these models via widely applicable information criterion (WAIC). The difference between the two models:

\section{$\Delta W A I C=$ WAIC $($ full model $)-W A I C($ model without term $)$,}

is reported in the Supplementary Information. Lower values of WAIC indicate better expected out-of-sample deviance. Accordingly, negative values of $\triangle$ WAIC indicate that a full model is preferred over the model with the term. Also, $\triangle$ WAIC was used to compute each model's relative weight $(W)$ reported in figures (Bürkner, 2018). The two weights add up to unity so that values above 0.5 indicate that the fixed factor's inclusion improves expected models' prediction accuracy. Finally, we compared models with and without a term via Bayes factor (BF) with values above $3.2,10$, and 100 indicating, respectively, substantial, strong, and decisive support for the model with the term (Kass \& Raftery, 1995).

For Experiment 1, we modelled the proportion of dominance time for individual perceptual states using beta family with a logit link and default priors (Bürkner, 2018). Specifically, we modelled the proportion of dominance times as:

$P_{\text {dominant }} \sim$ Stereo + Strength $+(1 \mid$ Participant $)$,

where $P_{\text {dominant }}$ is a proportion of time the perceptual state was dominant (values were scaled so that the range was from 0.0005 to 0.9995 to avoid extreme values of 0 and 1 ), Stereo $=$ Strength $>0^{\circ}$, Strength is the strength of disambiguation cues $\left(0^{\circ}, 0.25^{\circ}, 0.5^{\circ}, 1^{\circ}\right)$; Participant identity was used as a random factor.

For experiment 2, we modelled the number of trials when both spheres or each sphere rotated in the direction of the bias using the binomial family with a logit link and default priors 
(Bürkner, 2018):

$$
N_{\text {bias }} \mid N \sim \text { Strength }+ \text { Duration }+ \text { SOA }+ \text { Strength }
$$

: Duration $+(1 \mid$ Participant $)$,

where $N_{\text {bias }}$ is the number of trials when the sphere(s) rotated in the direction of the bias, $N$ - the total number of trials, Strength - the strength of disambiguation cues $\left(0^{\circ}, 0.25^{\circ}\right.$, $\left.0.5^{\circ}, 1^{\circ}\right)$, Duration - duration of the probe $(0.25 \mathrm{~s}, 0.5 \mathrm{~s}, 1 \mathrm{~s}$, $2 \mathrm{~s}), S O A$ - probe onset time relative to the onset time of the prime (0.7-0.9 s), Strength:Duration - an interaction term, Participant - random factor.

For Experiment 3, we assumed that the prime's influence on the probe depends on an overlap of two critical time windows: (1) an exponentially decaying strength of the switchinduced transient bias, following the on-screen-motion reversal event, (2) exponentially decaying sensitivity of the probe perception following its onset. The second time window corresponds to the process of perceptual inference until the probe's perception is resolved in favor of a particular state (Pastukhov, 2016). The prime's influence is proportional to an area of an overlap of the two curves (see Fig. 4a). Mathematically, we approximated this as:

$$
\begin{gathered}
\text { Prime Influence }=\exp \left(-\frac{T^{\text {later event }}-T^{\text {bias }}}{\tau_{\text {bias }}}-\frac{T^{\text {later event }}-T^{\text {probe }}}{\tau_{\text {probe }}}\right), \\
T_{\text {later event }}=\max \left(T^{\text {bias }}, T^{\text {probe }}\right),
\end{gathered}
$$

where $T^{\text {bias }}$ and $T^{\text {probe }}$ are, respectively, bias and probe onset time relative to the trigger event, $T^{\text {later event }}$ is a time of the later event in the trial, and $\tau^{\text {bias }}$ and $\tau^{\text {probe }}$ are, respectively, bias and probe decay time scales. We used independent bias onset and decay time constants for the two prime conditions, ambiguous and biased. We programmed a hierarchical Bayesian GLM in Stan (Carpenter et al., 2017) as follows:

$$
\begin{aligned}
& \begin{array}{c}
N_{i}^{\text {bias }} \sim \operatorname{Binomial}\left(N_{i}, p_{i}\right) \\
\operatorname{logit}\left(p_{i}\right)=\alpha_{P}+\beta_{P, C} \cdot \text { PrimeInfluence }\left(T_{P}^{\text {probe }}, T_{P, C}^{\text {bias }}, \tau_{P}^{\text {probe }}, \tau_{P, C}^{\text {bias }}\right)
\end{array} \\
& \alpha_{P} \sim \operatorname{Normal}\left(\mu^{\alpha}, \sigma^{\alpha}\right) \\
& \beta_{P, C} \sim \operatorname{Normal}\left(\mu_{C}^{\beta}, \sigma_{C}^{\beta}\right) \\
& T_{P, C}^{\text {bias }} \sim \operatorname{Normal}\left(\mu_{C}^{T_{\text {bias }}}, \sigma_{C}^{T_{\text {bias }}}\right) \\
& \tau_{P, C}^{\text {bias }} \sim \operatorname{Normal}\left(\mu_{C}^{\tau_{\text {bias }}}, \sigma_{C}^{\tau_{\text {bias }}}\right) \\
& \tau_{P}^{\text {probe }} \sim \operatorname{Normal}\left(\mu^{\tau_{\text {probe }}}, \sigma^{\tau_{\text {probe }}}\right) \\
& \mu_{C}^{T_{\text {bias }}} \sim \operatorname{Cauchy}(50,50) \\
& \mu_{C}^{\tau_{\text {bias }}}, \mu^{T_{\text {probe }}} \sim \text { HalfCauchy }(100,50) \\
& \mu^{\alpha}, \mu_{C}^{\beta} \sim \operatorname{Cauchy}(0,10) \\
& \sigma^{\alpha}, \sigma_{C}^{\beta}, \sigma_{C}^{T_{\text {bias }}}, \sigma_{C}^{\tau_{\text {bias }}}, \sigma^{\tau_{\text {probe }}} \sim \operatorname{HalfCauchy}(0,10),
\end{aligned}
$$

where the subscript $i$ indicates the data row, subscript $P$ is a participant index, subscript $C$ is a prime condition index (1biased, 2-ambiguous), $N^{\text {bias }}$ is the number of trials when a participant reported that the probe rotated in the direction of the bias, $N$ is the total number of trials, and $T^{\text {probe }}$ is the probe onset time relative to the on-screen motion reversal trigger event. Following McElreath (2016), we used weakly regularizing Cauchy and half Cauchy priors centered at zero for most parameters, except for $\mu_{C}^{T_{\text {bias }}}$ that was centered at $50(\mathrm{~ms})$ and $\mu_{C}^{\tau_{\text {bias }}}, \mu^{\tau_{\text {probe }}}$ that were centered at $100(\mathrm{~ms})$ based on prior work (Pastukhov, 2016). The model was sampled using four chains with parameters adapt_delta $=0.98$ and max_treedepth $=15$.

\section{Results}

\section{Experiment 1}

In our first experiment, we sought to replicate and extend earlier work on perceptual coupling, particularly the reduced perceptual coupling when one of the spheres is disambiguated (Grossmann \& Dobbins, 2003). To this end, we presented two rotating spheres that were placed either side-by-side (parallel axes of rotation; see Fig. 1a) or one above the other (coaxial layout, see Supplementary Materials for results). The rotation of one (prime) sphere was disambiguated via stereoscopic depth cues, whereas the other (probe) sphere was fully ambiguous. The participants viewed the spheres for one minute and continuously reported on the direction of rotation of both spheres.

Our results were in complete agreement with the earlier work, as the spheres were strongly perceptually coupled - rotated mostly in the same direction-when both were fully ambiguous (see Fig. 2a; see Fig. S1 and Table S1 in the Supplementary Materials). Also, as in the original report, the proportion of time the participants reported corotation decreased significantly for strongly disambiguated primes. However, the analysis showed that this effect was explained solely by a decrease in the proportion of time the two spheres rotated together against the bias. Instead, the proportion of time the two rotated together with the bias remained constant despite increasing strength of disambiguation cues (there was an effect of the mere presence of the depth cues but not of their strength; see Table S1 in the Supplementary Materials). For the individual spheres, even though stereoscopic depth cues significantly increased the proportion of time the prime sphere rotated in the direction of the bias, there was no significant effect on the ambiguous probe (see Fig. 2b). In short, increasing the amount of time the biased state was dominant for the prime sphere did not increase the dominance of that same state in the probe. This observation challenges the idea that perceptual coupling comes from a perceptually dominant state (Grossmann \& Dobbins, 2003). 


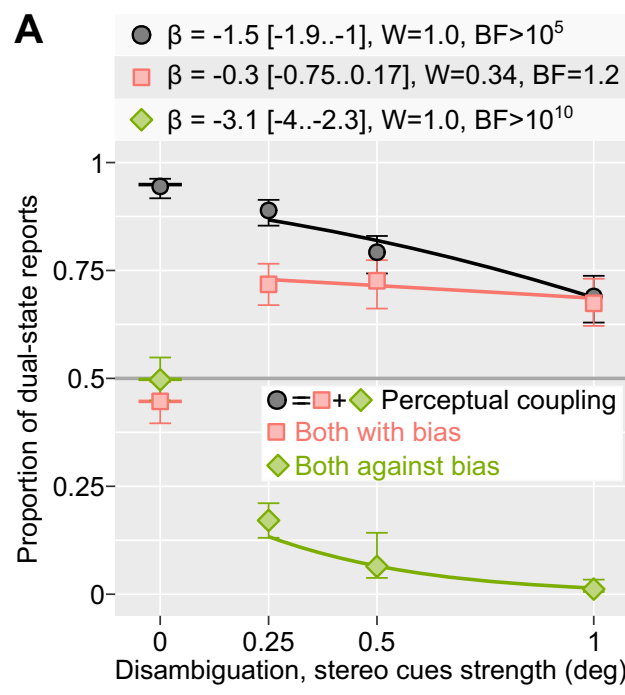

Fig. 2 Experiment 1. a The proportion of time the two spheres rotated in the same direction overall ( OPerceptual coupling), in the direction of the bias ( $\square$ Both with bias) or against the bias ( $\diamond$ Both against bias) as a function of stereo cues' strength. $\mathbf{b}$ The proportion of time the individual spheres ( $\odot$ Prime or $\square$ Probe) rotated in the direction of bias as a function of stereo cues' strength. a-b Circles and error bars depict group mean and bootstrapped 95\% bias-corrected accelerated confidence intervals. Solid lines show the prediction of a median model. The tables above the plot show the main effect of stereo

\section{Experiment 2}

To confirm that state-based mechanisms cannot explain perceptual coupling, we performed our second experiment that employed a brief asynchronous presentation of prime and probe spheres. We hypothesized that for state-based mechanisms, if a prime sphere appears before the probe, the dominant perceptual state of the former should perceptually couple the latter biasing its onset perceptual state towards the same direction of rotation. For this, we split the presentation sequence into pairs of trials (see Fig. 1b). During the first trial, the participants viewed and reported only on the ambiguous probe, informing us about the current dominant direction of rotation at that location (i.e., the direction of rotation we would expect to be dominant again at this location on the subsequent trial; Knapen et al., 2009). During the subsequent trial, the prime sphere appeared at the complementary position before the probe (stimulus onset asynchrony was 700-900 ms) and was biased to rotate in the opposite direction to that reported for the probe on the first trial (see Fig. 1b-d). The probe's delayed onset provided a perfect opportunity for bias from the prime's dominant perceptual state to influence it. Thus, if perceptual coupling reflects an influence of a dominant state, we would expect prime to reliably bias the onset perception of the probe towards its dominant direction (the probability that the probe rotated in the direction of bias $[P$ probe $=$ bias) $>0.5$ ]. Conversely, a tendency of the probe to rotate in the same direction as on the previous trial and, therefore,

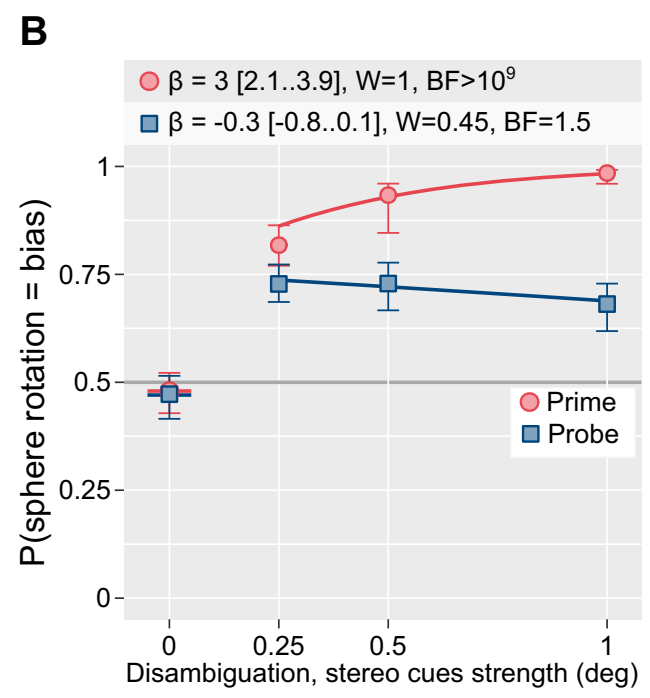

strength on each dual state's dominance (a) and direction rotation of a sphere (b); for further details, see Table S2 in the Supplementary Materials. $\beta=$ mean estimate and $95 \%$ credible interval; $\mathrm{W}=$ relative weight of the full model compared to a model without the stereo strength term (two weights add up to 1 , weight above 0.5 indicates the importance of the term); $\mathrm{BF}=$ Bayes factor for models with and without stereo strength term, value above 3.2, 10, and 100 indicating, respectively, substantial, strong, and decisive support for the model with the term. (Color figure online)

opposite to the prime $[P($ probe $=$ bias $)<0.5]$ would indicate a weak state-based perceptual coupling. We observed the latter, with uniformly low probabilities of the probe rotating in the direction of bias for all stimulus onset asynchronies and strength of the biasing cues (see Fig. 3). For the $\square$ Probe, the probability did decrease slightly for stronger disambiguation cues, but this effect was not statistically significant. In short, the prime's perceptual state had little influence on the perceptual state of the probe either during onset perception or continuous viewing (Experiment 1).

\section{Experiment 3}

The alternative to the state-based bias is a transient mechanism that is active whenever an object changes its perceptual state. This transient influence does not need to be strong to influence perceptual dominance if it occurs at the right time (i.e., at the stimulus onset; Song \& Yao, 2009) or when a currently dominant state is weakened by adaptation (Lankheet, 2006). To test this hypothesis, we replicated Experiment 2 using stable primes (as in Experiment 2) and extended it using switching primes. In the latter case, an on-screen motion reversal at a predefined time triggered a perceptual switch to the direction of rotation opposite to one reported for the probe on the previous trial. We hypothesized that if bias is transient and induced by a perpetual switch, we should see its effect only when the probe appears shortly before or after a perceptual switch (see Fig. 4a). Moreover, the attenuation of the prime's 


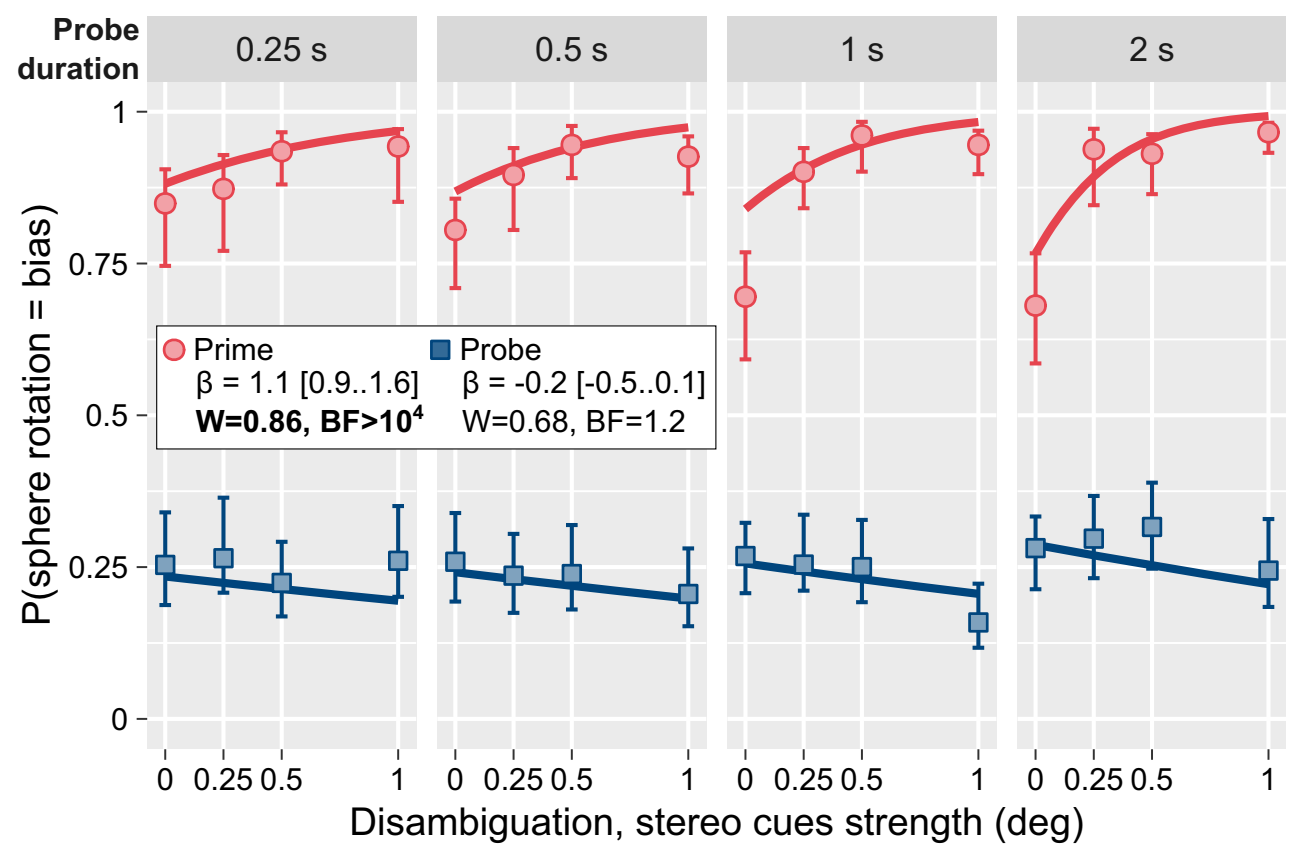

Fig. 3 Experiment 2 (see also Fig. S2 in the Supplementary Materials). The proportion of trials when an individual sphere ( $\bigcirc$ Prime or $\square$ Probe) rotated in the direction of bias and opposite to the direction of

influence on the probe should be proportional to decay times of both the transient bias and probe perception onset sensitivity.

The stable ambiguous prime condition replicated Experiment 2 and produced very similar results (see Fig. $4 \mathrm{~b}$, green line and diamonds). For the switching primes, both ambiguous (Fig. 4b, blue line and squares) and disambiguated (Fig. $4 b$, red line and circles), we found that perceptual switches biased the probe's perceptual state most strongly if it was presented shortly after the onscreen-motion reversal.

To quantify this effect, we fitted data using a hierarchical Bayesian model, assuming that the strength of the prime's influence reflects a degree of temporal overlap of the two exponentially decaying processes: 1) the transient changeinduced bias of the prime and 2) the onset sensitivity of the probe perception (see Fig. 4a and Methods for details). The fits for individual participants are shown in Fig. 4c. The model indicated that the transient bias occurs shortly after the onscreen motion-reversal event, with the perceptual switch itself occurring no earlier than $20 \mathrm{~ms}$ after that (Pastukhov \& Klanke, 2016), and decays rapidly (decay time constants 200-300 ms), see legend inset in Fig. 4c; see Fig. S3, and Table S3 in the Supplementay Materials. The bias was present for both ambiguous and unambiguous (disambiguated) primes but was weaker, occurred later, and decayed slower for the former. Thus, our results show that perceptual coupling works via a switch-induced mechanism that broadcasts a transient bias favoring the new perceptual state at nearby locations and objects. rotation for the probe reported on the previous trial (see Fig. 2 for figure and legend details, and Table S2, in the Supplementary Materials, for full statistical analysis). (Color figure on line)

\section{Discussion}

We reexamined the perceptual coupling phenomenon when several multistable displays tend to be in the same dominant perceptual state and switch synchronously. In Experiments 1 and 2, we ruled out a hypothesis that mutual bias reflects the influence of the perceptually dominant state as was proposed in the prior research (Grossmann \& Dobbins, 2003; Klink et al., 2009). Next, we demonstrated that the perception of bistable rotating spheres is influenced by a bias produced whenever one of them switches to a new dominant state. Moreover, we found that the transient bias was produced by perceptual switches in both ambiguous and disambiguated displays, showing that perceptual coupling is not specific to multistability and does not depend on stimulus ambiguity.

The mechanisms of change-detection play a crucial role in our daily lives (Simons \& Rensink, 2005), triggering reevaluation of perception (Serences \& Yantis, 2006), amplifying the change signal (Mehrpour et al., 2020), and ensuring, among other things, correct visual feature binding (Parto et al., 2021) and the possibility of rapid behavioral responses (Parto et al., 2021). Prior work indicated that these mechanisms are common to both unambiguous (Martinez-Trujillo et al., 2007) and multistable (Britz et al., 2009; Kanai et al., 2005; Kornmeier et al., 2009; Takahashi \& Watanabe, 2010) displays. Our results offer further support for this link and demonstrate how multistable perception can characterize temporal and spatial properties of these mechanisms. 

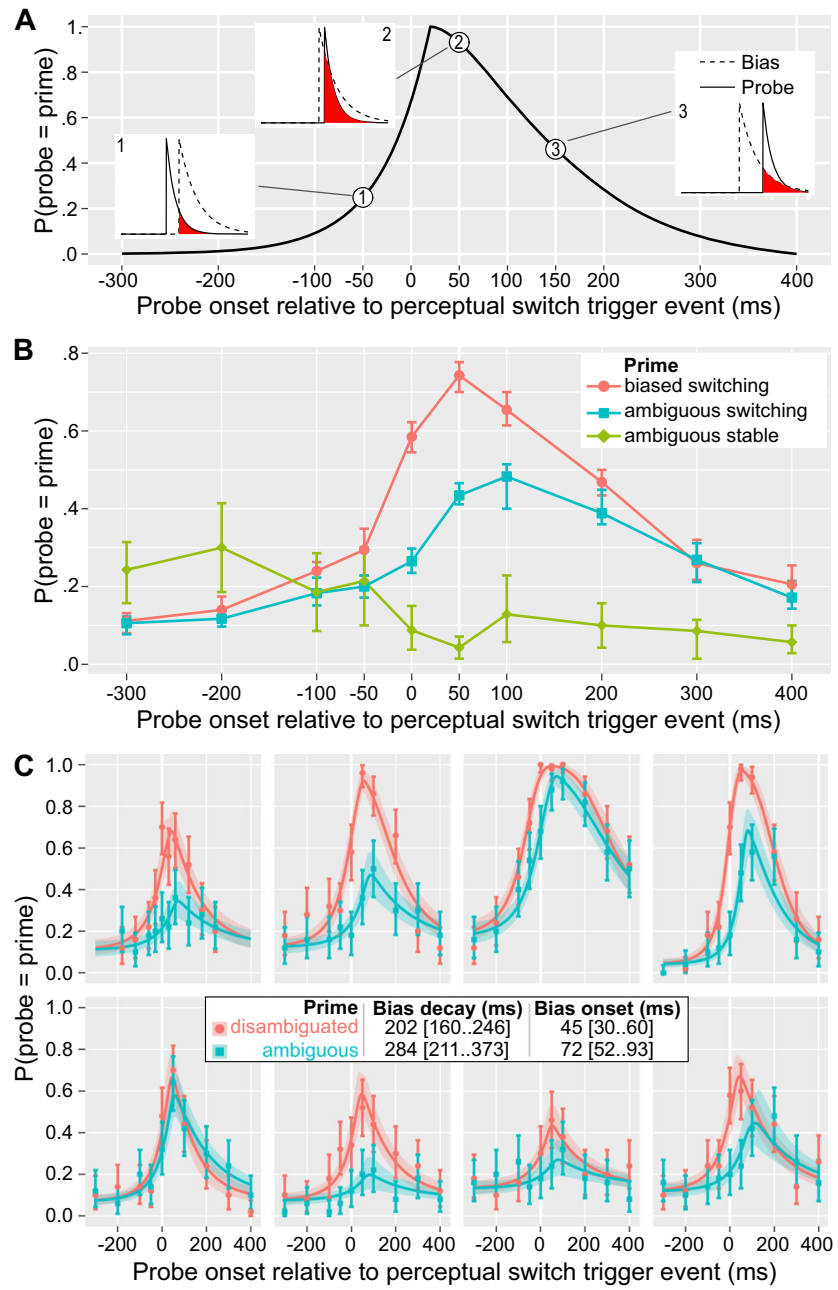

Fig. 4 Experiment 3. a Schematic model. An illustration of the interaction between exponentially decaying sensitivity of probe perception after the onset (solid line in inset plots, example $\tau_{\text {probe }}=50$ $\mathrm{ms}$ ) and exponentially decaying strength of the switched-induced bias (dashed line in inset plots, example $\tau_{\text {bias }}=100 \mathrm{~ms}$, bias onset $50 \mathrm{~ms}$ after the trigger event). The stronger influence of the prime on probe perception, reflected in higher $P($ probe $=$ prime $)$, corresponds to the larger overlap of the two time-windows (red area in inset plots). b Group average for the proportion of trials when the probe was reported to rotate in the same direction as the prime. Circles and error bars depict group mean and bootstrapped $95 \%$ bias-corrected accelerated confidence intervals, respectively. c Data and model fits for individual participants. The proportion of trials when the probe sphere rotated in the same direction as the prime, following the switch, and against the expected default rotation direction of the probe, as a function of the probe onset time relative to the on-screen-motion reversal event. Circles and error bars depict mean and bootstrapped $95 \%$ bias-corrected accelerated confidence intervals. Solid lines and stripes show the prediction of a median model and $95 \%$ credible interval. The legend inset show mean and 95\% credible intervals for population-level bias decay and onset parameters. (Color figure online)

\section{Switch-induced perceptual coupling}

Our results show that perceptual coupling is produced not by a dominant perceptual state, as was assumed in prior work (Grossmann \& Dobbins, 2003; Klink et al., 2009), but by a switch to a new dominant perception. This idea is fully compatible with prior experimental evidence and allows for a parsimonious explanation of observations that are problematic for a dominant-state bias hypothesis. As noted in the introduction, the latter has no simple explanation of why perceptual coupling is reduced when one of the objects is disambiguated (Grossmann \& Dobbins, 2003). Our results suggest that this is because rare perceptual switches in a disambiguated prime mean equally rare opportunities for it to influence probe via switch-induced transient bias. Conversely, the prime's high perceptual stability makes it resistant to transient bias produced by frequent perceptual switches in the probe. Thus, although the strength of the transient bias itself is not affected by sphere disambiguation, the combination of these two factors leads to an apparent decrease of perceptual coupling during continuous viewing.

Another problematic finding is that perceptually coupled displays tend to switch more frequently than a single sphere (Pastukhov et al., 2018). The dominant-state bias hypothesis predicts longer dominance phases because the mutual stabilizing bias should increase the dominant state's relative strength, biasing perception in its favor. In contrast, the switch-induced transient bias predicts observed shorter dominance durations for coupled displays because either object can initiate a joint perceptual switch, doubling the sources of perceptual instability.

In short, an idea of switch-induced transient bias offers a more parsimonious account for accumulated experimental evidence than a dominant state bias theory.

\section{Perceptual consequences of endogenous and exogenous perceptual switches}

Perceptual switches produced the transient bias in both ambiguous and disambiguated spheres meaning that it is common to endogenous (spontaneous) and exogenous (physical) perceptual switches. The difference between the two is one of the critical questions in multistability research. On the one hand, prior research identified transient neural activity in certain frontal and parietal areas specific to spontaneous but not perceptually matched physical switches (Brascamp et al., 2018) but see (Kornmeier \& Bach, 2012). On the other hand, the same meta-analysis revealed transient activity common to both types of switches in other areas associated with perceptual decision making and feedback mechanisms, such as the dorsolateral prefrontal cortex. Recent work by de Jong et al. (2020), using the intracranial recording, demonstrated that endogenous and exogenous switches rely on the same perceptual hierarchy, differing primarily in the order that these areas are activated.

Our results highlight another commonality between exogenously and endogenously induced switches by 
showing that both trigger the same mechanism of perceptual reevaluation. Taken together, this suggests that although spontaneous and physical switches may differ in how they are initiated, they tap into the same or shared processing networks and lead to qualitatively and, even, quantitatively similar perceptual consequences.

\section{Possible neural mechanisms of switch-induced tran- sient bias}

Prior research suggested top-down feedback as a possible mechanism for perceptual coupling (Eby et al., 1989; Grossmann \& Dobbins, 2003). Selective attention, in particular, would be a prime candidate. It modulates both rates of perceptual switches (Pastukhov \& Braun, 2007) and strength of perceptual coupling (Mareschal \& Clifford, 2012), although it is not strictly necessary for either to occur (but see Dieter et al., 2016, on a special case of binocular rivalry). In this framework, a perceptual switch could activate a "circuit breaker" ventral frontoparietal network (Corbetta \& Shulman, 2002), attracting attention and prompting a reevaluation of perception to reestablish coherent activity across regions of the visual system (Serences \& Yantis, 2006). Here, a transient feedback signal would stabilize a new perceptual state (de Jong et al., 2020; Weilnhammer et al., 2013), affecting spatially adjusted populations with similar selectivity. The "spilling over" effect would reflect a larger receptive field size of neurons at the top of the processing cascade (Dumoulin \& Wandell, 2008) and could be an integral part of perceptual inference over space (Spillmann et al., 2015).

Alternatively, a prior work modelled perceptual coupling via local lateral connections between similarly and oppositely tuned groups of neurons (Klink et al., 2009). Although this model assumed sustained bias, it is likely to be similarly capable with a switch-induced transient bias. Similarly, a model of binocular rivalry for ring displays, which is essentially a perceptual coupling display composed of multiple adjacent binocular rivalry stimuli, allowed for traveling waves of dominance using just a two-layer model with only local connectivity (Lee et al., 2005). These mechanisms could be like those that facilitate rapid contour integration in the primary visual cortex (Pack \& Born, 2001; VanRullen et al., 2001). In the end, although we lean towards the selective attention hypothesis, it is up to future research to resolve this question.

\section{Conclusions}

To conclude, we report that both endogenous and exogenous perceptual switches produce a transient signal that initiates perceptual reevaluation in the nearby locations. It is likely to reflect transient top-down feedback that stabilizes the new perceptual state of the changed object and biases nearby locations towards the new dominant state.

Supplementary Information The online version contains supplementary material available at https://doi.org/10.3758/s13423-021-01960-7.

Funding Open Access funding enabled and organized by Projekt DEAL.

Declarations All procedures were in accordance with the national ethical standards on human experimentation and with the Declaration of Helsinki of 1975 , as revised in 2008, and were approved by the University of Bamberg via the local Ethic committee-Der Ethikrat der Otto-Friedrich-Universität Bamberg.

Disclosure statement We report no potential conflicts of interest.

Open Access This article is licensed under a Creative Commons Attribution 4.0 International License, which permits use, sharing, adaptation, distribution and reproduction in any medium or format, as long as you give appropriate credit to the original author(s) and the source, provide a link to the Creative Commons licence, and indicate if changes were made. The images or other third party material in this article are included in the article's Creative Commons licence, unless indicated otherwise in a credit line to the material. If material is not included in the article's Creative Commons licence and your intended use is not permitted by statutory regulation or exceeds the permitted use, you will need to obtain permission directly from the copyright holder. To view a copy of this licence, visit http://creativecommons.org/licenses/by/4.0/.

\section{References}

Attneave, F. (1968). Triangles as Ambiguous Figures. The American Journal of Psychology, 81(3), 447. https://doi.org/10.2307/1420645

Brascamp, J. W., Sterzer, P., Blake, R., \& Knapen, T. H. J. (2018). Multistable perception and the role of the frontoparietal cortex in perceptual inference. Annual Review of Psychology, 69(1), 77-103. https://doi.org/10.1146/annurev-psych-010417-085944

Britz, J., Landis, T., \& Michel, C. M. (2009). Right parietal brain activity precedes perceptual alternation of bistable stimuli. Cerebral Cortex (New York, N.Y. : 1991), 19(1), 55-65. https://doi.org/10.1093/ cercor/bhn056

Bürkner, P.-C. (2018). Advanced Bayesian multilevel modeling with the $\mathrm{R}$ package brms. The $R$ Journal, 10(1), 395. https://doi.org/10. 32614/RJ-2018-017

Carpenter, B., Gelman, A., Hoffman, M. D., Lee, D., Goodrich, B., Betancourt, M., Brubaker, M., Guo, J., Li, P., \& Riddell, A. (2017). Stan: A probabilistic programming language. Journal of Statistical Software, 76(1). https://doi.org/10.18637/jss.v076.i01

Corbetta, M., \& Shulman, G. L. (2002). Control of goal-directed and stimulus-driven attention in the brain. Nature Reviews Neuroscience, 3(3), 201-215. https://doi.org/10.1038/nrn755

de Jong, M. C., Vansteensel, M. J., van Ee, R., Leijten, F. S. S., Ramsey, N. F., Dijkerman, H. C., Dumoulin, S. O., \& Knapen, T. H. J. (2020). Intracranial Recordings Reveal Unique Shape and Timing of Responses in Human Visual Cortex during Illusory Visual Events. Current Biology, 30(16), 3089-3100.e4. https://doi.org/10. 1016/j.cub.2020.05.082 
Dieter, K. C., Brascamp, J. W., Tadin, D., \& Blake, R. (2016). Does visual attention drive the dynamics of bistable perception? Attention, Perception, \& Psychophysics, 78(7), 1861-1873. https:// doi.org/10.3758/s13414-016-1143-2

Dumoulin, S. O., \& Wandell, B. A. (2008). Population receptive field estimates in human visual cortex. NeuroImage, 39(2), 647-660. https://doi.org/10.1016/j.neuroimage.2007.09.034

Eby, D. W., Loomis, J. M., \& Solomon, E. M. (1989). Perceptual linkage of multiple objects rotating in depth. Perception, 18(4), 427-444. https://doi.org/10.1068/p180427

Fang, F., \& He, S. (2004). Stabilized structure from motion without disparity induces disparity adaptation. Current Biology: CB, 14(3), 247-251. https://doi.org/10.1016/j.cub.2004.01.031

Freeman, E. D., \& Driver, J. (2006). Subjective appearance of ambiguous structure-from-motion can be driven by objective switches of a separate less ambiguous context. Vision Research, 46(23), 4007-4023. https://doi.org/10.1016/j.visres.2006.07.008

Gibson, J. J., \& Radner, M. (1937). Adaptation, after-effect and contrast in the perception of tilted lines: I. Quantitative studies. Journal of Experimental Psychology, 20(5), 453-467. https://doi.org/10.1037/ h0059826

Grossmann, J. K., \& Dobbins, A. C. (2003). Differential ambiguity reduces grouping of metastable objects. Vision Research, 43(4), 359369. https://doi.org/10.1016/S0042-6989(02)00480-7

Hansen, T., Walter, S., \& Gegenfurtner, K. R. (2007). Effects of spatial and temporal context on color categories and color constancy. Journal of Vision, 7(4), 2-2. https://doi.org/10.1167/7.4.2

Intaite, M., Noreika, V., Šoliunas, A., \& Falter, C. M. (2013). Interaction of bottom-up and top-down processes in the perception of ambiguous figures. Vision Research, 89, 24-31. https://doi.org/10.1016/j. visres.2013.06.011

Kanai, R., Moradi, F., Shimojo, S., \& Verstraten, F. A. J. (2005). Perceptual alternation induced by visual transients. Perception, 34(7), 803-822. https://doi.org/10.1068/p5245

Kass, R. E., \& Raftery, A. E. (1995). Bayes factors. Journal of the American Statistical Association, 90(430), 773-795. https://doi. org/10.1080/01621459.1995.10476572

Klink, P. C., Noest, A. J., Holten, V., van den Berg, A. V., \& van Wezel, R. J. A. (2009). Occlusion-related lateral connections stabilize kinetic depth stimuli through perceptual coupling. Journal of Vision, 9(10), 20-20. https://doi.org/10.1167/9.10.20

Klink, P. C., van Wezel, R. J. A., \& van Ee, R. (2012). United we sense, divided we fail: context-driven perception of ambiguous visual stimuli. Philosophical Transactions of the Royal Society B: Biological Sciences, 367(1591), 932-941. https://doi.org/10.1098/rstb.2011. 0358

Knapen, T. H. J., Brascamp, J. W., Adams, W. J., \& Graf, E. W. (2009). The spatial scale of perceptual memory in ambiguous figure perception. Journal of Vision, 9(13), 16.1-12. https://doi.org/10.1167/9.13. 16

Kornmeier, J., \& Bach, M. (2012). Ambiguous digures-What happens in the brain when perception changes but not the stimulus. Frontiers in Human Neuroscience, 6(March), 1-23. https://doi.org/10.3389/ fnhum.2012.00051

Kornmeier, J., Hein, C. M., \& Bach, M. (2009). Multistable perception: When bottom-up and top-down coincide. Brain and Cognition, 69(1), 138-147. https://doi.org/10.1016/j.bandc.2008.06.005

Lankheet, M. J. M. (2006). Unraveling adaptation and mutual inhibition in perceptual rivalry. Journal of Vision, 6(4), 1. https://doi.org/10. $1167 / 6.4 .1$
Lee, S.-H., Blake, R., \& Heeger, D. J. (2005). Traveling waves of activity in primary visual cortex during binocular rivalry. Nature Neuroscience, 8(1), 22-23. https://doi.org/10.1038/nn1365

Mareschal, I., \& Clifford, C. W. G. (2012). Perceptual entrainment of individually unambiguous motions. Journal of Vision, 12(1), 1-8. https://doi.org/10.1167/12.1.24

Martinez-Trujillo, J. C., Cheyne, D., Gaetz, W., Simine, E., \& Tsotsos, J. K. (2007). Activation of area MT/V5 and the right inferior parietal cortex during the discrimination of transient direction changes in translational motion. Cerebral Cortex, 17(7), 1733-1739. https:// doi.org/10.1093/cercor/bhl084

McElreath, R. (2016). Statistical rethinking. Chapman and Hall/CRC. https://doi.org/10.1201/9781315372495

Mehrpour, V., Martinez-Trujillo, J. C., \& Treue, S. (2020). Attention amplifies neural representations of changes in sensory input at the expense of perceptual accuracy. Nature Communications, 11(1), Article 2128. https://doi.org/10.1038/s41467-020-15989-0

Murray, S. O., Boyaci, H., \& Kersten, D. (2006). The representation of perceived angular size in human primary visual cortex. Nature Neuroscience, 9(3), 429-434. https://doi.org/10.1038/nn1641

Pack, C. C., \& Born, R. T. (2001). Temporal dynamics of a neural solution to the aperture problem in visual area MT of macaque brain. Nature, 409(6823), 1040-1042. https://doi.org/10.1038/35059085

Parto, M., Schwedhelm, P., Wibral, M., Treue, S., Reza, M., \& Esghaei, M. (2021). A neural correlate of visual feature binding in primate lateral prefrontal cortex. NeuroImage, 229(January), Article 117757. https://doi.org/10.1016/j.neuroimage.2021.117757

Pastukhov, A. (2016). Perception and the strongest sensory memory trace of multi-stable displays both form shortly after the stimulus onset. Attention, Perception, \& Psychophysics, 78(2), 674-684. https://doi. org/10.3758/s13414-015-1004-4

Pastukhov, A., \& Braun, J. (2007). Perceptual reversals need no prompting by attention. Journal of Vision, 7(10), Article 5. https:// doi.org/10.1167/7.10.5

Pastukhov, A., \& Klanke, J.-N. (2016). Exogenously triggered perceptual switches in multistable structure-from-motion occur in the absence of visual awareness. Journal of Vision, 16(3), Article 14. https://doi. org $/ 10.1167 / 16.3 .14$

Pastukhov, A., Vonau, V., \& Braun, J. (2012). Believable change: Bistable reversals are governed by physical plausibility. Journal of Vision, 12(1), 17-17. https://doi.org/10.1167/12.1.17

Pastukhov, A., Zaus, C. R., Aleshin, S., Braun, J., \& Carbon, C.-C. (2018). Perceptual coupling induces co-rotation and speeds up alternations in adjacent bi-stable structure-from-motion objects. Journal of Vision, 18(4), Article 21. https://doi.org/10.1167/18.4.21

Peirce, J., Gray, J. R., Simpson, S., MacAskill, M., Höchenberger, R., Sogo, H., Kastman, E., \& Lindeløv, J. K. (2019). PsychoPy2: Experiments in behavior made easy. Behavior Research Methods, 51(1), 195-203. https://doi.org/10.3758/s13428-018-01193-y

R Core Team. (2019). R: A Language and Environment for Statistical Computing. https://www.r-project.org/

Ramachandran, V. S., \& Anstis, S. M. (1983). Perceptual organization in moving patterns. Nature, 304(5926), 529-531. https://doi.org/10. 1038/scientificamerican0686-102

Ramachandran, V. S., \& Anstis, S. M. (1985). Perceptual organization in multistable apparent motion. Perception, 14(2), 135-143. https:// doi.org/10.1068/p140135

Serences, J. T., \& Yantis, S. (2006). Selective visual attention and perceptual coherence. Trends in Cognitive Sciences, 10(1), 38-45. https://doi.org/10.1016/j.tics.2005.11.008

Sereno, M. E., \& Sereno, M. I. (1999). 2-D center-surround effects on 3D structure-from-motion. Journal of Experimental Psychology: 
Human Perception and Performance, 25(6), 1834-1854. https://doi. org/10.1037/0096-1523.25.6.1834

Simons, D. J., \& Rensink, R. A. (2005). Change blindness: Past, present, and future. Trends in Cognitive Sciences, 9(1), 16-20. https://doi. org/10.1016/j.tics.2004.11.006

Song, C., \& Yao, H. (2009). Duality in binocular rivalry: distinct sensitivity of percept sequence and percept duration to imbalance between monocular stimuli. PLOS ONE, 4(9), Article e6912. https:// doi.org/10.1371/journal.pone.0006912

Spillmann, L., Dresp-Langley, B., \& Tseng, C. (2015). Beyond the classical receptive field: The effect of contextual stimuli. Journal of Vision, 15(9), Article 7. https://doi.org/10.1167/15.9.7

Takahashi, K., \& Watanabe, K. (2010). Implicit auditory modulation on the temporal characteristics of perceptual alternation in visual competition. Journal of Vision, 10(4), 1-13. https://doi.org/10.1167/10. 4.11

Todorović, D. (2010). Context effects in visual perception and their explanations. Review of Psychology, 17(1), 17-32. https://hrcak.srce. $\mathrm{hr} / 70655$
VanRullen, R., Delorme, A., \& Thorpe, S. (2001). Feed-forward contour integration in primary visual cortex based on asynchronous spike propagation. Neurocomputing, 38-40, 1003-1009. https://doi.org/ 10.1016/S0925-2312(01)00445-3

Weilnhammer, V. A., Ludwig, K., Hesselmann, G., \& Sterzer, P. (2013). Frontoparietal cortex mediates perceptual transitions in bistable perception. The Journal of Neuroscience: The Official Journal of the Society for Neuroscience, 33(40), 16009-16015. https://doi.org/10. 1523/JNEUROSCI.1418-13.2013

Wickham, H., Averick, M., Bryan, J., Chang, W., McGowan, L., François, R., Grolemund, G., Hayes, A., Henry, L., Hester, J., Kuhn, M., Pedersen, T., Miller, E., Bache, S., Müller, K., Ooms, J., Robinson, D., Seidel, D., Spinu, V., Takahashi, K., Vaughan, D., Wilkek K., Woo, K., \& Yutani, H. (2019). Welcome to the Tidyverse. Journal of Open Source Software, 4(43), Article 1686. https://doi.org/10.21105/joss.01686

Publisher's note Springer Nature remains neutral with regard to jurisdictional claims in published maps and institutional affiliations. 